\title{
Knowledge Driven Orbit-to-Ground Teleoperation of a Robot Coworker
}

\author{
P. Schmaus, D. Leidner, T. Krüger, R. Bayer, B. Pleintinger, A. Schiele, and N. Y. Lii
}

\begin{abstract}
The crewed exploration of Moon and Mars requires the construction and maintenance of infrastructure on the alien surfaces before a crew arrives. Robotic coworkers are envisioned to take over the physical labor required to set-up crew habitats, energy supplies, and return vehicles in the hazardous environment. Deploying these robots in such a remote location poses a challenge that requires autonomous robot capabilities in combination with effective Human Robot Interfaces (HRIs), which comply with the harsh conditions of deep space operations. An astronaut-robot teleoperation concept targeting these topics has been evaluated in DLR and ESA's METERON SUPVIS Justin experiment where astronauts on-board the International Space Station (ISS) commanded DLR's humanoid robot Rollin' Justin in a simulated Martian environment on Earth. This work extends on our previously presented approach to supervised autonomy. It examines the results of the two follow-up experiment sessions which investigated maintenance and assembly tasks in real-world scenarios. We discuss the use of our system in real space-toground deployment and analyze key performance metrics of the HRI and the feedback given by the astronauts.
\end{abstract}

Index Terms-Space Robotics and Automation, Telerobotics and Teleoperation, Robotics in Hazardous Fields

\section{INTRODUCTION}

$\mathbf{T}$ HE Global Exploration Roadmap compiled by the International Space Exploration Coordination Group states that crewed exploration of the surface of Moon and Mars are the common driving goals of the space agencies worldwide [1]. Future missions to these extraterrestrial destinations raise a greatly heightened need for robots to support the astronauts with setting up and maintaining infrastructure on the surface before and after the crew's arrival. These robots would be equipped with autonomous capabilities as direct teleoperation from Earth becomes unfeasible with increasing communication time delays. An approach to reduce the communication delay and thus enable more efficient teleoperation is to command the surface robots from an orbiting spacecraft. Due to the limited crew time, as many tasks as possible should be delegated to the autonomous capabilities of the robot, whereas the astronaut should only intervene in exceptional situations.

The robot command interface operated by the astronauts must be designed to account for the specific requirements on

Manuscript received: June 6, 2019; Revised: August 23, 2019; Accepted: October 15, 2019. This paper was recommended for publication by J. Roberts upon evaluation of the Associate Editor and Reviewers' comments.

P. Schmaus, D. Leidner, R. Bayer, B. Pleintinger, and N. Y. Lii are with the Institute of Robotics and Mechatronics, German Aerospace Center (DLR), Wessling, Germany; T. Krüger is with the Human Robot Interaction Lab, European Space Agency (ESA), Noordwijk, The Netherlands; A. Schiele is with the Faculty of Mechanical, Materials and Maritime Engineering, Delft University of Technology, Delft, The Netherlands. peter.schmaus@dlr. de Preprint Version

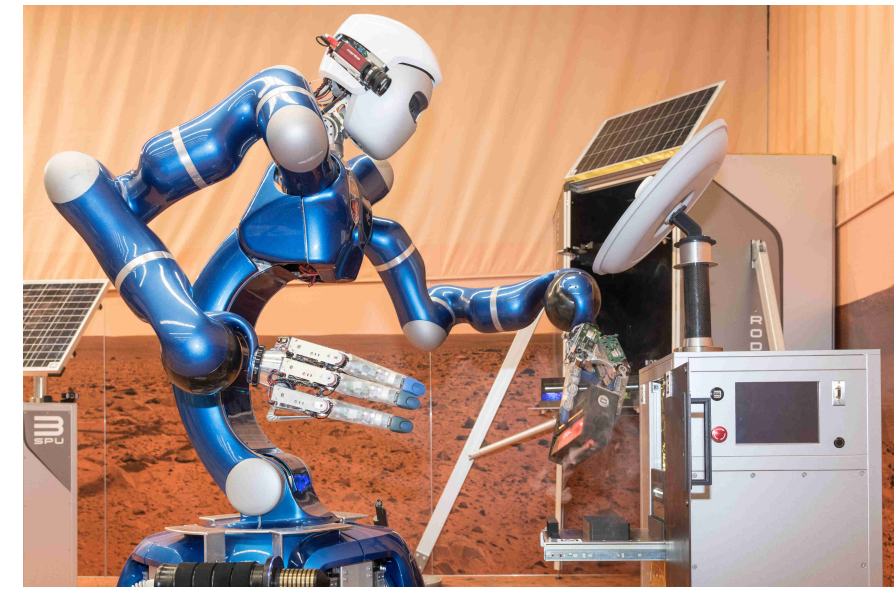

Fig. 1. DLR's Rollin' Justin replacing a defective component as commanded by the ISS crew during the METERON SUPVIS Justin experiment.

an orbiting spacecraft. Microgravity can alter the astronauts' motor skills and the operation of a vessel in deep space brings high mental load [2]. In addition, the orbital communication link with the robot on the surface is limited in terms of variable delay, jitter, signal loss, and bandwidth. Using the robot's local intelligence to take over lower level tasks has been shown to be effective in terrestrial applications [3]. Extending on this taskspace approach, we proposed a supervised autonomy approach for robot commanding that lets the astronaut use the taskspecific intelligence of the robot [4]. This makes the robot an intelligent coworker rather than a tool for the astronaut, efficiently reducing the mental and physical workload for the astronaut compared to the direct teleoperation of the robot.

This work builds on our prior work on a knowledge driven approach for effective teleoperation of an intelligent service robot from an orbiting spacecraft [4]. The contributions in this work include $(i)$ extensions to the approach to allow command parameterization and pinning, (ii) an on-ground evaluation by a user study, and (iii) comparative on-orbit evaluation with trained and untrained astronauts on board the ISS, and $(i v)$ commanding maintenance and assembly tasks in real-world scenarios. The results discussed in this paper provide the space robotics community with an assessment of the possibilities offered by this key technology for the future construction of infrastructures on celestial bodies, as depicted in Fig. 1.

The remainder of this paper is structured as follows: Section II gives an overview of the related work with an focus on crewcentric surface telerobotics. Our astronaut-robot collaboration concept is detailed in Section III and evaluated in Section IV. 
Section V describes the on-orbit experiments conducted using the proposed user interface. The results of the experiments are discussed in Section VI. This paper concludes in Section VII with an outlook on future missions and the transfer of the findings to on-ground applications.

\section{RELATED WORK}

According to the Global Exploration Roadmap, robots deployed on Moon and Mars would be used to explore the environment, collect and analyze samples, deploy instruments, prepare landing sites, and setup and maintain infrastructure for future (crewed) missions [1]. To maximize the use of these surface assets, researchers have proposed for the astronauts to command surface robots from on-board orbiting spacecrafts during future crewed missions [5][6][7][8].

As real orbit-to-ground experiments are rarely available, researchers evaluate approaches for robotic teleoperation in analogue experiments. These experiments often aim to mimic the extraterrestrial environment of the robot (e.g. [9], [10]) or challenging communication links (e.g. [11], [12]). The remainder of this section gives an overview of past orbit-toground teleoperation experiments.

In 2009, the Canadian Space Agency (CSA) performed the Avatar-EXPLORE experiment for testing a planetary rover navigating autonomously under infrequent supervision [13]. The astronaut on-board the ISS received and analyzed telemetry files from the rover, generated a command sequence, and prepared a corresponding command file that was downlinked to the rover for execution. Six robot command sequences were completed in three hours experiment time, showing that the proposed approach is suitable for commanding semiautonomous rovers with infrequent supervision. An important aspect observed during the experiment was that the astronaut developed trust in the rover's autonomy leading to more efficient utilization of the unit over time.

NASA's 2012-2014 Surface Telerobotics experiments simulated a lunar mission with astronauts on-board an orbiter and a rover on the surface [14]. An interactive approach has been tested for commanding the rover using discrete and supervisory commands for navigation and inspection via a communication link with an average delay of $500-750 \mathrm{~ms}$. In three sessions of 3.5 hours each, the astronauts commanded the rover to (1) survey the site and identify a target location, (2) deploy a radio telescope, and (3) inspect the deployed asset. The use of safeguarding capabilities of the rover in combination with interactive $3 \mathrm{D}$ visualizations reduced the cognitive load of the astronaut while keeping the robot in a safe state. The experiment demonstrated the efficiency of supervisory command with interactive monitoring [15].

In 2015-2017, DLR and Roscosmos investigated a telepresence approach for crewed command of surface robots in the Kontur-2 project [16][17]. A 2-Degree Of Freedom (DOF) force-feedback joystick for commanding surface robots from microgravity was evaluated in 33 ISS sessions. A direct station-to-ground communication link enables a short latency of $20-30 \mathrm{~ms}$. This requires the ISS to be in the range of the horizon, with a session duration of 8-10 minutes. These experiments demonstrated that telepresent force-feedback command

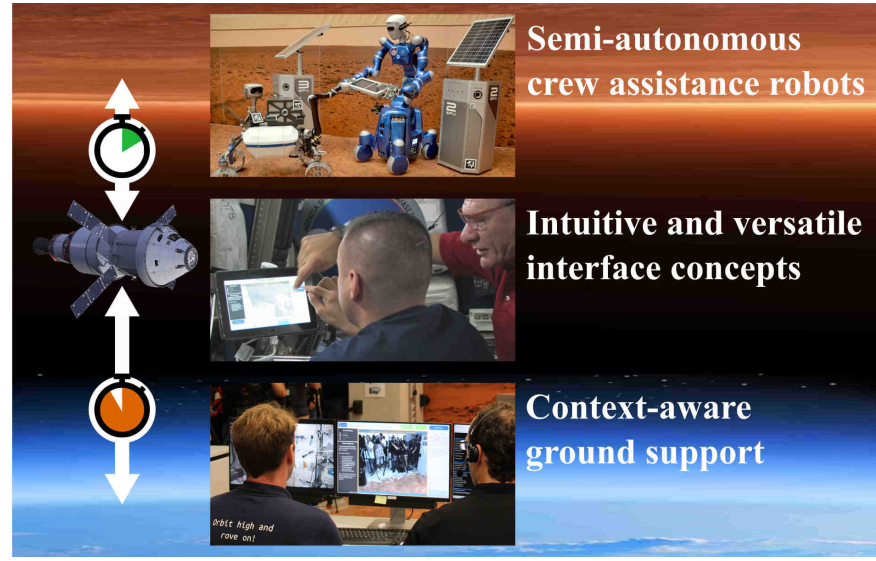

Fig. 2. Overview of the proposed astronaut-robot collaboration concept for future planetary research from an orbiting spacecraft.

is efficient for deploying robots for prior unknown situations, requiring physical contact of the robot with unknown objects, when a communication link with a delay below 1 s and minimal jitter is available.

ESA initiated the Multi-Purpose End-To-End Robotic Operation Network (METERON) project together with DLR, NASA, and Roscosmos [18] to understand the operation and relevant technology of space telerobotics. The METERON HAPTICS experiments investigated the human perception of force-feedback in microgravity [19][20]. The UI hardware consisting of a 1-DOF force-feedback joystick and a tablet computer was upmassed to the ISS in 2014. During the HAPTICS experiments, various studies have been conducted with ISS astronauts, such as commanding a surface robot via a communication link with a latency of $800 \mathrm{~ms}$. In the METERON Interact experiment, an astronaut on-board the ISS operated a robot at the European Space research and TEchnology Centre (ESTEC) to execute a force-feedbackteleoperated sub-millimeter precision peg-in-hole task [21]. To reduce the workload of the operator, the commands for approaching the manipulation target were based on the semiautonomous capabilities of the robot, and transferred to the HRI by the use of visual assistance markers. The markers augmented the live video feed of the robot with predictive information of the pending robot navigation commands.

The SUPVIS-E and SUPVIS-M experiments started the investigation of supervisory robot command in METERON by utilizing predefined task-level commands [22]. The astronauts used the METERON Operations Software Graphical User Interface (GUI) installed on a laptop computer to issue commands and monitor the autonomous execution during the ISS sessions [23]. The primary finding of the experiments was that task-level commanding of a remote robot is highly effective for task executing via a limited communication link.

Although all the described approaches have been used to successfully command the respective robots in the respective scenarios, none presented a scalable HRI approach suited for a wide variety of robots and real-world maintenance and assembly scenarios. Furthermore, the described approaches employed robot-centered commands and exhaustive telemetry 
displays that require the operator to be familiar with the commanded robot [24]. In this work, we describe a different HRI concept that exploits the intelligence of the robot as main anchor point of the interface to overcome these limitations.

\section{Astronaut-Robot TELEOPERATION CONCEPT}

In our previous work, we introduced an HRI concept that utilizes the autonomous capabilities of the robot to provide the operator with an intuitive interface [4]. In this section, we give a quick overview of the system, and propose extensions to allow the operator to command more complex tasks not solvable by the robot alone. An overview of the proposed system is depicted in Fig. 2.

\section{A. Semi-Autonomous Crew Assistance Robot}

In previous work we have shown the advantages of organizing the knowledge of the robot in an object-centered context [25]. The information required for the robot to understand its environment and manipulate objects, is stored with the objects. A central object storage holds the knowledge and can be shared between different robots. The robots use the information from the object storage to reason about the environment and create a World Representation that holds the current state of the environment by the means of object instances with specific geometric and symbolic properties.

The object functionality is provided via Action Templates, which consist of a symbolic header and a geometric body. We use the symbolic reasoning of the robot to determine all actions that are currently feasible for later use in the HRI [26]. With this approach, we are able to realize knowledge driven human-robot interaction. The actions determined in this way consist of a unique name, the symbolical effects of execution, and a set of parameters that specify the objects concerned and the action-specific execution options. For better guidance of the astronaut towards task completion, the list of possible actions is context-specifically updated on the fly, as described in the following subsection.

\section{B. Context-Aware Ground Support}

Depending on the current World Representation of the robot, the generated list of actions can be quite extensive (e.g. if there are many objects with various Action Templates around the robot). There may also be actions available not needed by the astronaut to accomplish the task at hand. In order to unclutter the information passed on to the astronaut, we implemented a utility for context-specific pruning of the list of possible actions. Therefore, the complexity of each action is rated according to the number of additional actions that need to be executed to resolve the desired effects. Geometric relations between objects are explicitly modeled. This information is used for applying symbolic, geometric, and context-specific filters to the list of possible actions. The filters are managed by a Mission Control Center (MCC) (located on ground), which enables precise tuning of the astronaut's command options for task- and user-specific guidance [27]. The resulting list of actions is the basis for commanding the robot, as detailed below.

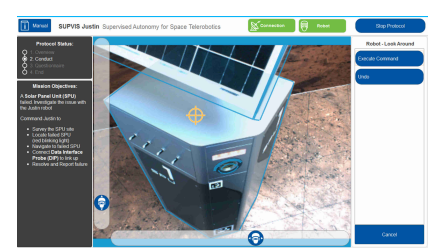

(a)

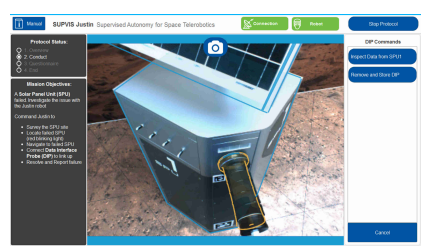

(b)
Fig. 3. HRI implemented for METERON SUPVIS Justin: The camera direction (pan/tilt) is commanded by positioning a crosshair (a), and selecting a virtually augmented object accesses the object-related command options (b). For best view, please refer to a digital version of the article.

\section{Intuitive and Versatile User Interface}

With an autonomous robot serving as a coworker, astronauts could manage the robot as a side task. This calls for interface hardware such as portable devices that can be used in parallel with other activities. Tablet computers have a proving track record as effective UI, and provide a form factor and capability well suited for semi-autonomous robot commanding, as we have shown in previous work [26]. To be approved for use onboard the ISS, the look-and-feel of the GUI has to comply with the ISS Display and Graphics Commonality Standard [28].

A key to telerobotic mission success is the astronaut's awareness of the remote environment of the robot. This poses a challenge as the small tablet screen limits the information available for the astronaut. As we utilize the robot's autonomy for low-level safeguards, we establish a common ground between the robot and the astronaut by displaying the live video of the robot. The video is augmented with CAD renderings of the objects of the robot's current World Representation, as visible in Fig. 3. The astronaut can easily assess the precision of the robot calibration or object detection, by reviewing the match of the edges of rendering and the objects in the video.

The basis for the concrete commanding of the robot is the list of context-specific reasonable robot commands, described in section III-B. This enables our HRI approach to be independent of the concrete robotic system or scenario, as it relies only on the commands generated online by the generic autonomy system. This paves the way for future experiments, in which we aim to command multiple robots using the same interface. To further reduce the amount of displayed information to the commands of immediate interest, we cluster the commands w.r.t. the affected objects and bind the generated objectspecific command lists to the augmented 3D objects. The visible objects in the video become interactive GUI elements that can be selected using a point-and-click approach. This effectively reduces robot commanding to selecting the object the astronaut wants to interact with, followed by selecting an object-specific command from a short list. By commanding the robot on this high level of abstraction, the requirements on the communication link are lowered compared to traditional teleoperation (e.g. telepresence), since only distinct command/response packets need to be sent without real-time requirements as the robot autonomously ensures a safe state. This allows us to use the HRI with communication links with high latency and frequent signal losses. After the robot finished the command processing, the astronaut can correct possible execution errors by issuing respective robot commands. 


\section{System Extension}

Robot commands may provide a set of parameters for tuning the effects to the corresponding Action Templates, e.g. the target orientation when rotating a solar panel. In the initial version of the HRI, presented in [4], the robot autonomously decided on the parameterization of the command based on the current state of robot and environment. The upgraded HRI adds the functionality for the astronaut to manually adjust these parameters to guide the task execution of the robot. Therefore the robot first evaluates valid parameter options or ranges, which can then then be specified by the operator in parameter selection GUI dialogues using sliders or drop-down menus. The implementation of command-specific parameterization GUIs simplifies the parameter selection for the astronaut, e.g. by augmenting the target orientation of the solar panel in the robot's video.

For easier access of often used commands, favorite commands can be pinned to the top of the commands list by long pressing the desired command.

The presented supervised autonomy HRI allows the astronaut to be in charge of the mission, as a supervisor commanding the robotic coworker. We implemented the concept for the use on-board of the ISS and evaluated the approach prior to the deployment in space.

\section{Evaluation}

The applicability of the HRI concept described in Section III is demonstrated for the use on-board the ISS. Therefore we setup a system for on-ground evaluation that can be transparently connected to the ISS communication link for subsequent space experiment sessions.

\section{A. Implementation}

The surface robot used in our experiments is Rollin' Justin, a humanoid robot developed at DLR for research in the field of service robotics [29]. Advanced time-invariant wholebody control strategies allow the robot precise and compliant interaction with its environment even when commanded via an unreliable communication link [30].

The robot is located in the SOLar farm EXperimental space robotics validation (SOLEX) environment located at DLR Oberpfaffenhofen, Germany (see Fig. 4) [31]. SOLEX is a simulated Martian solar farm environment for the testing and evaluation of future space service robots and provides the RObex DemonstratIon laNder (RODIN) mock-up and three Smart Payload Units (SPUs) used to test a variety of servicing tasks. The SPUs can be equipped with different payloads such as solar panels, antennas, or computation units. RODIN provides SPU spare parts for robotic servicing tasks and operates as communication hub for Rollin' Justin, the SPUs, and the MCC. The SOLEX environment also served as the proving ground to evaluate the HRI concept through a user study.

The HRI tablet computer is connected to the RODIN lander via a simulated space communication link for the onground validation experiments that can later be transparently replaced by the real communication link to the ISS. For the

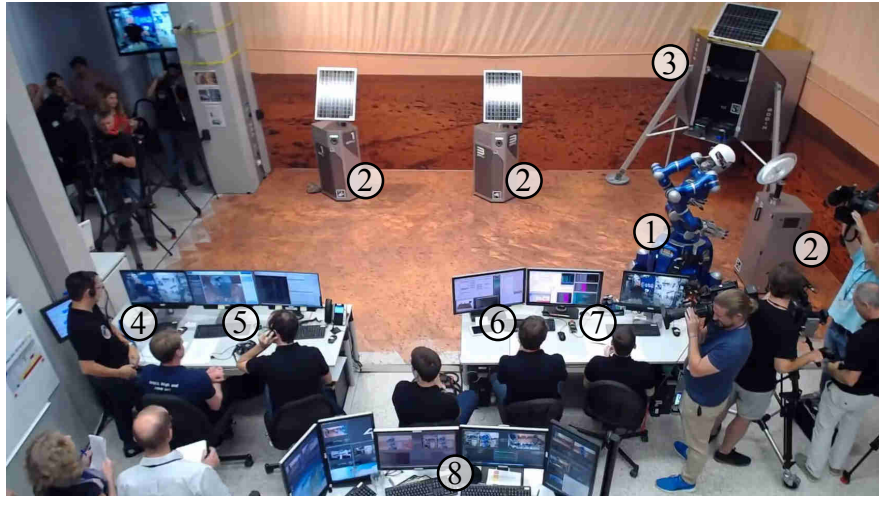

Fig. 4. Top view of the laboratory setup including (1) Rollin' Justin, (2) Smart Payload Units, (3) RODIN lander mockup, (4) PI console for direct communication with the astronauts, (5) operations and telemetry console, (6) robot console, (7) mission control display, and (8) data logging console.

validation experiments, we simulated a communication link with a limited bandwidth of $1 \mathrm{Mbit} / \mathrm{s}$ up- and down-link and a latency of $800 \mathrm{~ms}$. In addition, Loss-Of-Signal (LOS) events could be scheduled to account for possible communication interruptions when communicating with the ISS.

Our interface concept can be realized on most generic tablet devices. For SUPVIS Justin, we implemented the HRI application for the use on the tablet computer used in the METERON HAPTICS experiments.

\section{B. On-Ground User Study}

We carried out the individual user study session following the same procedures as planned for the ISS sessions to be conducted by the crew members in space operations conditions. Accordingly, the test participant was placed in a booth isolated from SOLEX and the MCC after having a short familiarization with Rollin' Justin and its environment. The communication channel between the HRI and the robot was set-up to simulate the expected space-link with a roundtrip delay of $800 \mathrm{~ms}$ and a maximum bandwidth of $1 \mathrm{Mbit} / \mathrm{s}$ for up- and downlink. In addition, communication with the study investigators in the Ground Control Center was only possible, via an audio channel similar to the Voice Communication Subsystem (VoCS) used to communicate with astronauts on-board the ISS [32].

The study was conducted with 7 female and 13 male participants between the ages of 21 and 55 (average 29.6). All the participants were familiar with tablet computers but not with the examined HRI or Rollin' Justin. Twelve of the participants were familiar with other robotic systems and were recruited from the surrounding of the lab. The user study sessions were each carried out for a contiguous 120 minutes, emulating the experiment protocol portion of each ISS session. The first ten minutes were allocated for study concept explanation without using the HRI yet or detailing on the tasks of the study or the features of the HRI. A total of three tasks with different levels of complexity and task nature were prepared for the participants: (T1) find and restart a defective SPU, (T2) install an antenna on a SPU and replace a defective computation unit, (T3) optimize the solar panel efficiency of a SPU by readjusting and cleaning the panel. 


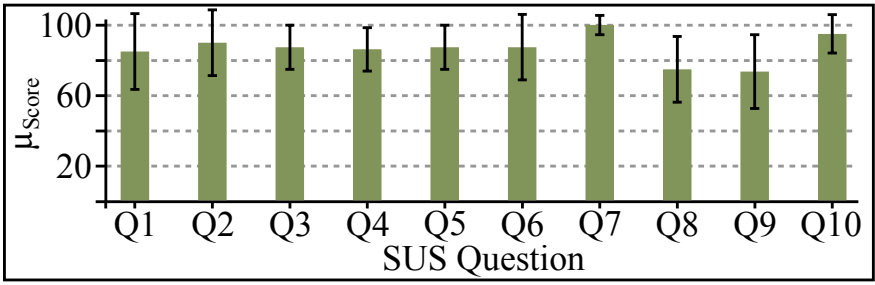

Fig. 5. Results of the user study SUS questionnaire. For more information about the standardized SUS questions refer to [33].

After a task has been completed, the participants answered a questionnaire regarding environmental awareness and workload during the study, and general feedback on the HRI. The respective questions were carefully formulated to allow for insights in the system usage while avoiding ESA medical board examination. This approach simplifies the recruitment of ISS crew members during the real space to ground verification sessions. After each user study session concluded, an additional System Usability Scale (SUS) [33] questionnaire has been completed in order to measure the usability of the HRI on a scale from 0 (bad) to 100 (good).

All participants succeeded in commanding the robot to complete T1 and T2. Only 14 of the participants could also complete $\mathrm{T} 3$ due to the time constraint. In spite of this, the responses to the questionnaire were consistently positive. All participants quickly became familiar with the supervised autonomy approach, within the first of three protocols. They also expressed positive feedback, particularly for its ease-ofuse and scalability to handle a larger team of robots for larger and more complex tasks. The capability of the UI to provide situational awareness in the robot's environment was rated $8.62 / 10$ in the questionnaire, although the robot's limited field of view was deemed to be the factor that slowed down work progress the most. The context specific task guidance of the UI was rated 8.03/10 for helping to understand the situation and complete the task at hand.

The SUS results depicted in Fig. 5 showed a high overall score of $\mu 85.5$ ( $\sigma$ 5.73). The most room for improvement has been reported in terms of confidence in the system $\left(\mu_{\mathrm{Q} 9} 72.5\right)$ and its cumbersomeness $\left(\mu_{\mathrm{Q} 8}\right.$ 73.75). This is particularly due to the small screen size of the tablet PC placing a limitation on the amount of information displayed. However, with increasing experience, the score improved as participants gained more confidence in the robot's autonomy system. Very high scores were obtained in terms of intuitiveness ( $\mu_{\mathrm{Q} 7}$ 93.75) and easy learning ( $\mu_{\mathrm{Q} 10}$ 98.75).

The results from the user study demonstrated that the proposed system is well suited to command a remote surface robot. Accordingly, on-orbit experiments with astronauts onboard the ISS were conducted to verify the approach with operators in a realistic environment.

\section{SPACE-TO-GRound ExPERIMENT}

In the METERON SUPVIS Justin experiment DLR and ESA evaluated the intuitive supervised autonomy HRI presented in this work [31][34]. By performing real on-orbit experiments, we demonstrated the operational readiness of

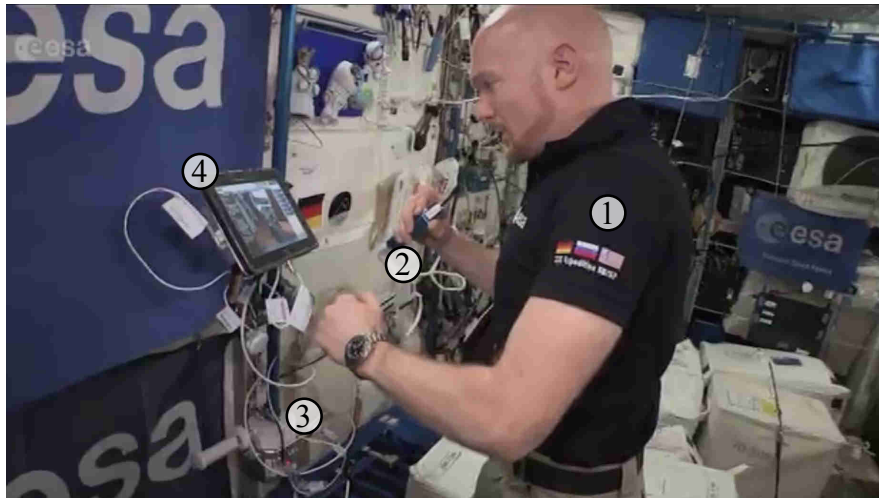

Fig. 6. SUPVIS Justin ISS setup including (1) ESA astronaut Alexander Gerst performing the experiment, (2) the microphone for voice communication with ground control, (3) the METERON HAPTICS 1-DOF joystick, and (4) the HRI application running on the tablet computer.

the lightweight HRI for commanding semi-autonomous robots from orbit with minimal mental load for the operator.

\section{A. Experiment Setup}

In the experiment, we used the components described in Section IV-A, in particular Rollin' Justin as a surface service robot and SOLEX as Martian analogue site.

METERON SUPVIS Justin utilizes a tablet computer and communication gateway previously upmassed for the METERON HAPTICS experiments (see Fig. 6) [19]. We were able to connect to the ISS LAN through the communication gateway, creating a bidirectional communication link to SOLEX via various NASA and ESA facilities using ESAs Multi Purpose Computer and Communication software suite. A detailed overview of the space communication link is given by [4]. The METERON SUPVIS Justin experiment marked the first time that such a connection from an ISS payload system was used to command a semi-autonomous service robot on Earth. In this work, we expand upon our previous paper on commanding inspection tasks [4] to the more complex tasks of maintenance and assembly in real-world scenarios.

\section{B. ISS Sessions}

The on-orbit evaluation of the system was carried out in three ISS experiment sessions that built upon each other. Each session lasted 4 hours, including setup and stowage of the HAPTICS kit. After each session, we analyzed the feedback of the astronaut, MCC, and associated projected partners to further improve the system. In order to keep the results of the different sessions comparable, only minor changes to the UI were implemented as requested by the astronaut operators: user-definable favorite commands, a larger view of the robots' camera view, and an automatic display of the camera view while executing a command.

Prior to their spaceflights, the selected astronauts performed a training at ESAs Astronaut Training Centre in Cologne to prepare for the experiment. During this course, they received an introduction to the telerobotic system and SOLEX environment as well as instructions for setting-up the HRI hardware on-board the ISS. 
The first ISS experiment session was conducted on August 25th, 2017. Five different experiment scenarios were completed by three different astronauts, two of whom were trained in-flight. During the session, the astronauts commanded Rollin' Justin to perform a variety of inspection and simple manipulation tasks which gave us affirmation of the ease of use of our approach, as well as the effectiveness of onthe-fly crew-to-crew training [4]. The results of the experiment session assured us that the HRI could also be used for more complex scenarios.

On March 2nd 2018, the second ISS session of METERON SUPVIS Justin was conducted. In addition to the scenarios from the first experiment, a different astronaut also commanded Rollin' Justin to optimize the efficiency of a solar panel. Therefore, the crew member commanded the robot to navigate and physically connect to a SPU, download the current status data, and determine the efficiency of the solar panel. Subsequently, commands to iteratively optimize the efficiency were issued for panel cleaning, and panel reorientation with available fine adjustment inputs. The astronaut finally succeeded in finding a maximum efficient orientation of the panel. The experiment session result demonstrated that the situational and task awareness via our HRI is sufficient for more complex scenarios.

The final ISS experiment session of METERON SUPVIS Justin has been conducted on August 17th 2018. The subject on-board the ISS first completed the reference scenario from the first ISS session before commanding the robot to retrieve an antenna from RODIN and install it on top of a target SPU. After the successful hardware installation, we simulated a fatal error for one of the Computation Units inside the SPU for this experiment. The subject successfully identified the problem and removed the damaged part in time from the SPU to avoid further damage. The astronaut then installed a spare Computation Unit from RODIN in the defective SPU to bring it back online. In the remaining time, the subject succeeded to complete the solar panel optimization task introduced in the second experiment session, allowing us to gather more data on manual command parameterization and optimization tasks.

\section{RESUlTS AND DiscusSiON}

Extending on the first METERON SUPVIS Justin experiment presented in [4], this work demonstrates an increased level of task complexity, with the first successful execution of maintenance and assembly tasks on a planetary surface in a real-world scenario commanded by astronauts in orbit. We measured an average communication roundtrip delay of 832ms (min: 800ms, max: 1132ms) and handled multiple planned LOS events inherent to the communication link used during the ISS-to-ground experiment sessions. The semiautonomous operation kept the robot in a safe state throughout the experiment and is able to cope with more challenging communication conditions with multi-second delay and higher jitter as expected for lunar missions.

The experimental results have shown that the autonomy of the robot can be used to realize an intuitive knowledge driven HRI that reduces the cognitive load on astronauts. The

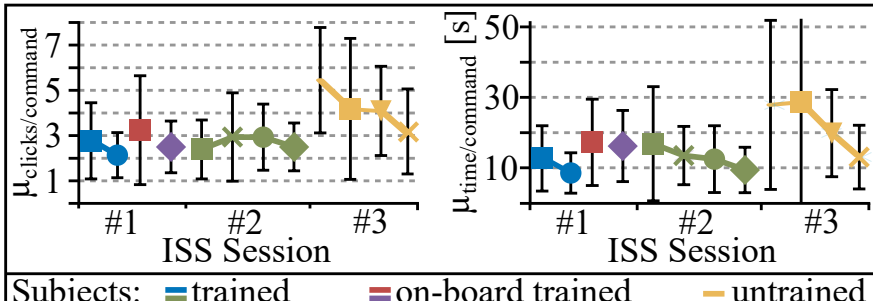

Scenario: $\quad$ reboot $\bigcirc$ firmware $>$ sandstorm $\times$ clean $\nabla$ install

Fig. 7. UI usage data in the space-to-ground sessions: Mean clicks (left) and mean time per robot command (right) for each experiment scenario.

command statistics shown in Fig. 7 visualize the familiarization of the astronauts with the system as the clicks and time needed to issue a command decrease with their experience. The decreasing standard-deviations shown in Fig. 7 indicate increasing confidence in issuing commands as the astronaut quickly become familiar with the setup.

A key feature for intuitive operation of our HRI was the augmentation of the live video with $3 \mathrm{D}$ objects of the World Representation of the robot. With this feature, the astronauts could estimate the status of the remote robot with low cognitive effort resulting in very good score range ( $\mu$ 8.42/10) for situational awareness in the evaluation questionnaire. The feedback of the astronauts suggests that this score could be further improved by increasing the field of view or provide more range of motion for reorientation of the robots' camera. Furthermore, a clearer visual separation of the augmented 3D objects improves the ease of use of the HRI, particularly for distinguishing overlapped objects and cluttered environments. By using the augmented 3D objects as dynamic GUI elements for accessing the context-specifically relevant commands of the robot, we demonstrated an intuitive approach for hiding the complexity of robot commanding while guiding the astronaut towards task completion.

Our key to providing effective, and easy to follow user guidance lies in providing only reasonable commands to the astronauts that are gathered by the symbolic reasoning of the robot and the task-specific filtering of the MCC. This approach is implemented in two GUI components: (1) The interaction with the augmented 3D objects in the live video allows the operator focus on the objects the robot should interact with to complete the current task; (2) the context specific task display shows an live updated summary of the current task with important indicators towards task completion. The astronauts would assess the task guidance through these two components sufficient to render traditional step-by-step protocol descriptions unnecessary. This resulted in near perfect questionnaire scores in terms of task awareness ( $\mu$ 9.64/10). The astronauts confirmed this assessment by successfully completing scenarios in which they had to correctly apply robot commands that were unknown to them before the experiment.

One of the subjects received only basic informational briefing without actual on-device training of the HRI prior to his spaceflight. During the ISS experiment session, the subject received an introduction to the GUI before conducting the reference scenario and some additional guidance from the 
MCC at the start of the session. The task completion time of this subject was $136 \%$ longer in the reference scenario than the fully trained astronauts, and $51 \%$ longer than the crew members trained on-board the ISS. We believe the main cause to be the subject's need to become acclimated to the direct selection of the augmented 3D objects in combination with the context-specific command availability. After issuing the first commands in this fashion, the subject quickly understood the concept and then attained similar performance times to the other astronaut participants. The vast differences in ease of task command, and completion time, between the trained astronauts versus the untrained, highlights the importance of proper training to effective task completion - even for an intuitive HRI. On the other hand, this may also point to the need for better designed Earth-to-space training instructions, so that such situations can be more effectively covered.

An unforeseen challenge we observed during the ISS sessions was the perceived ambiguity of some command names by some astronauts. These are usually due to the differences in interpretations of technical or operational terminology due to the dissimilar vocational backgrounds. One of the subjects had difficulty understanding the Localize command, used for determining the pose of a target object, because a similar naming has been used in another robotics experiment for a different functionality. This highlights the issue that the understanding of the command names strongly depend on the previous experience of the operator in robot commanding and raises the need for more extensive command descriptions for future experiments that can optionally be accessed by the astronaut at any time.

We observed that a favorite commands pane enables more efficient speedy task execution by comparing task completion times and the number of commands for task completion. During Session 1 - with favorite commands - 20 commands were issued to complete the reference protocol in 603s. Compared to this baseline, the astronaut of Session 2 needed $50 \%$ more commands (30) and $25.2 \%$ more time (806s) to finish the same protocol without favorite commands available in the UI. As a result, a user-defined favorite command pane was reintroduced in Session 3 with the goal of improving the task effectiveness. Nevertheless, the task completion time remained higher (701s) due to the untrained astronaut executing the protocol.

The astronauts highly rated $(\mu$ 8.4/10) our robotic teleoperation approach for commanding future surface robots, particularly for known and repetitive tasks. The effective information reduction employed in the HRI allows the use of small devices like tablet computers enabling the astronaut to conduct robot commanding tasks anywhere as a side-task. By utilizing the autonomous capabilities of the system, the remote robot is treated more as a coworker rather than a tool resulting in reduced cognitive load for the astronaut ( $\mu$ 2.24/10). The low cognitive workload of the astronauts also manifested in their assessment to be able to command the robot in more complex scenarios or to command a team of multiple heterogeneous robots at the same time.

Although the astronauts valued and enjoyed the low workload caused by the proposed HRI, they nonetheless still expressed the desire to be able to command the robot on higher and lower levels of autonomy. Higher level of autonomy commands allows the robot to execute combinations of actions to speed-up task completion and further lower the cognitive load on the operator. These commands are desirable for wellknown task sequences that have shown to be error resistant. In contrast to traditional task sequence robot commanding, the robot should autonomously determine and execute the required task steps. In contrast, lower level of autonomy commands allow the operator to take command of basic robot movements as e.g. Cartesian position of the end effector. This command mode increases the workload while enabling the operator to resolve situations the robot cannot solve autonomously. The decision as to which level of autonomy is appropriate for the respective task should be left to the astronaut, who can adapt the autonomy according to the current task requirements. The investigation of HRIs supporting robot commanding on different levels of autonomy while providing various input modalities is subject of future work.

\section{CONCLUSION AND FUTURE WORK}

Within the METERON SUPVIS Justin ISS sessions, we demonstrated the viability of our knowledge driven HRI for commanding remote semi-autonomous surface robots. By integrating the robots' knowledge of the environment into an intuitive HRI, we demonstrated that even small UI devices, such as tablet computers, can be used to effectively command remote robots. We confirmed the applicability of our approach in both simple and complex scenarios and validated the low cognitive workload for the astronaut operators in orbit.

In future space robotics experiments, we plan to focus on adding further input modalities to our HRI and exploring a sliding autonomy approach where the operator can define the robot's level of autonomy. Furthermore, we will continue investigating the commanding of a robotic coworker as a side task in parallel to other work in combination with the commanding of a heterogeneous team of robots with variable communication delays.

Another focus of our future work is to demonstrate the scalability of our approach for a wide variety of terrestrial applications. Therefore we adapted the presented HRI to command personal service robots in the household settings in the home robotic assistance project, SMiLE [35]. In the project, service robots support people in need of care such as physically handicapped or senior citizens.

METERON SUPVIS Justin now serves as the most advanced and closest facsimile to an actual human-robot team mission of orbit-to-ground collaboration around a celestial body. In addition to the real spacecraft conditions of the ISS, the stress of working in the confined space, the pressure of limited available time, as well as the pressure to perform the experiment in front of a large global public, all contribute to simulating real future mission. These make the user data we collected an invaluable foundation for developing future UI for an astronaut to effectively command a team of intelligent surface robots. 


\section{ACKNOWLEDGMENT}

We thank our astronauts participating in the ISS sessions for valuable insights and discussions: Paolo Nespoli, Jack Fischer, Randy Bresnik, Scott Tingle and Alexander Gerst.

Implementing the ISS communication link would not have been possible without the support of the German Space Operations Center (GSOC) and the Columbus Coltrol Centre $(\mathrm{Col}-\mathrm{CC})$. Special thanks goes to European Astronaut Training Centre and Danish Aerospace for their support during experiment preparation, testing, training, and execution.

\section{REFERENCES}

[1] International Space Exploration Coordination Group, "The Global Exploration Roadmap," www.globalspaceexploration.org, January 2018.

[2] B. Weber, S. Schätzle, C. Riecke, B. Brunner, S. Tarassenko, J. Artigas, R. Balachandran, and A. Albu-Schäffer, "Weight and Weightlessness Effects on Sensorimotor Performance During Manual Tracking," in Proceedings of the 10th International Conference on Haptics: Perception, Devices, Control, and Applications, vol. 9774. Berlin, Heidelberg: Springer-Verlag, 2016, pp. 111-121.

[3] N. Y. Lii, Z. Chen, M. A. Roa, . Maier, B. Pleintinger, and C. Borst, "Toward a Task Space Framework for Gesture Commanded Telemanipulation," in IEEE International Workshop on Robot-Human Interactive Communication (Ro-Man), 2012.

[4] P. Schmaus, D. Leidner, T. Krüger, A. Schiele, B. Pleintinger, R. Bayer, and N. Y. Lii, "Preliminary Insights From the METERON SUPVIS Justin Space-Robotics Experiment," IEEE Robotics and Automation Letters, vol. 3, no. 4, pp. 3836-3843, 2018.

[5] J. Burns, D. Kring, J. Hopkins, S. Norris, T. Lazio, and J. Kasper, "A lunar L2-Farside exploration and science mission concept with the Orion Multi-Purpose Crew Vehicle and a teleoperated lander/rover," Advances in Space Research, vol. 52, pp. 306-320, 2013.

[6] W. Carey, P. Schoonejans, B. Hufenbach, K. Nergaard, F. Bosquillon de Frescheville, J. Grenouilleau, and A. Schiele, "METERON: A mission concept proposal for preparation of human-robotic exploration," in Proceedings of the IAF/AIAA Global Space Exploration Conference. International Astronautical Federation, 2012.

[7] D. Lester, K. Hodges, C. Ower, and K. Klaus, "Exploration telepresence from Earth-Moon Lagrange points," in Proceedings of the IAF/AIAA Global Space Exploration Conference. International Astronautical Federation, 2012.

[8] S. Oleson, G. Landis, M. McGuire, and G. R. Schmidt, "HERRO Mission to Mars using Telerobotic Surface Exploration from Orbit," vol. 64, pp. 304-313, 092011.

[9] A. Wedler, M. Vayugundla, H. Lehner, P. Lehner, M. Schuster, S. Brunner, W. Stürzl, A. Dömel, H. Gmeiner, B. Vodermayer et al., "First Results of the ROBEX Analogue Mission Campaign: Robotic Deployment of Seismic Networksfor Future Lunar Missions," in Proc. of the IAF International Astronautical Congress (IAC), 2017.

[10] L. Graham, T. G. Graff, R. A. Yingst, L. Inge, and P. Russell, "2012 Moon Mars Analog Mission Activities on Mauna Kea, Hawai' i," Advances in Space Research, vol. 55, no. 10, pp. 2405-2413, 2015.

[11] A. K. Tanwani and S. Calinon, "A Generative Model for Intention Recognition and Manipulation Assistance in Teleoperation," in Proc. of the IEEE/RSJ International Conference on Intelligent Robots and Systems (IROS), 2017, pp. 43-50.

[12] M. V. Jakuba, C. R. German, A. D. Bowen, L. L. Whitcomb, K. Hand, A. Branch, S. Chien, and C. McFarland, "Teleoperation and Robotics under Ice: Implications for Planetary Exploration," in Proc. of the IEEE Aerospace Conference, 2018, pp. 1-14.

[13] E. Dupuis, P. Langlois, J. L. Bedwani, D. Gingras, A. Salerno, P. Allard, S. Gemme, R. L'Archevêque, and T. Lamarche, " The Avatar-EXPLORE experiments: Results and lessons learned," in Proceedings of the International Symposium on Artificial Intelligence, Robotics, and Automation in Space, 2010.

[14] M. Bualat, M. Deans, T. W. Fong, C. Provencher, D. Schreckenghost, and E. Smith, "ISS Crew Control of Surface Telerobots," in Proceedings of the IAF/AIAA Global Space Exploration Conference. International Astronautical Federation, 2012
[15] M. Bualat, T. Fong, M. Allan, X. Bouyssounouse, T. Cohen, L. Flückiger, R. Gogna, L. Kobayashi, Y. Lee, S. Lee, C. Provencher, E. Smith, V. To, H. Utz, D. Wheeler et al., "Surface Telerobotics: Development and Testing of a Crew Controlled Planetary Rover System," in Proceedings of the AIAA SPACE Conference, 092013.

[16] J. Artigas, R. Balachandran, C. Riecke, M. Stelzer, B. Weber, J.-H. Ryu, and A. Albu-Schaeffer, "KONTUR-2: Force-Feedback Teleoperation from the International Space Station," in IEEE International Conference on Robotics and Automation (ICRA), 2016.

[17] M. Stelzer, B. M. Steinmetz, P. Birkenkampf, J. Vogel, B. Brunner, and S. Kühne, "Software Architecture and Design of the Kontur-2 Mission," in IEEE Aerospace Conference, 2017, pp. 1-17.

[18] A. Schiele, "METERON - Validating Orbit-to-Ground Telerobotics Operations Technologies," in 11th Symposium on Advanced Space Technologies for Robotics and Automation (ASTRA), 2011.

[19] A. Schiele, M. Aiple, T. Krueger, F. van der Hulst, S. Kimmer, J. Smisek, and E. den Exter, "Haptics-1: Preliminary Results from the First Stiffness JND Identification Experiment in Space," in Proc. of the International Conference on Human Haptic Sensing and Touch Enabled Computer Applications. Springer, 2016, pp. 13-22.

[20] A. Schiele, T. Krüger, S. Kimmer, M. Aiple, J. Rebelo, J. Smisek, E. den Exter, E. Mattheson, A. Hernandez, and F. van der Hulst, "Haptics-2 - A System for Bilateral Control Experiments from Space to Ground via Geosynchronous Satellites," in IEEE International Conference on Systems, Man, and Cybernetics (SMC). IEEE, 2016.

[21] A. Schiele, "Towards the Interact Space Experiment: Controlling an Outdoor Robot on Earth's Surface from Space," in Proc. of the 13th Symposium on Advanced Space Technologies for Robotics and Automation (ASTRA), 2015.

[22] M. Sarkarati, M. Merri, K. Nergaard, and P. Steele, "How to plugin your rover into a space mission to moon or mars," in Automation, Robotics and Applications (ICARA), 2015 6th International Conference on. IEEE, 2015, pp. 318-324.

[23] M. Cardone, C. Laroque, M. Sarkarati, K. Nergaard, P. Steele, and S. Martin, "MOE: A System Infrastructure for Robotic Experiments," in Space Operations: Contributions from the Global Community. Springer, 2017, pp. 27-52.

[24] S. Hart, J. Kramer, S. Gee, and R. R. Burridge, "The PHARAOH Procedure Execution Architecture for Autonomous Robots or Collaborative Human-Robot Teams," in IEEE International Symposium on Robot and Human Interactive Communication, 2016, pp. 888-895.

[25] D. S. Leidner, Cognitive Reasoning for Compliant Robot Manipulation. Springer, 2019.

[26] P. Birkenkampf, D. Leidner, and C. Borst, "A Knowledge-Driven Shared Autonomy Human-Robot Interface for Tablet Computers," in IEEE/RAS International Conference on Humanoid Robots (Humanoids). IEEE, 2014, pp. 152-159.

[27] D. Leidner, P. Birkenkampf, and N. Y. Lii, "Context-aware Mission Control for Astronaut-Robot Collaboration," in 14th Symposium on Advanced Space Technologies in Robotics and Automation, 2017.

[28] National Aeronautics and Space Administration, "Display and Graphics Commonality Standard," http://spaceflight.esa.int/eo/EOI/crew-in fosys/bkgnd/SSP50313RevD.pdf, SSP 50313, Accessed: 2019-08-22.

[29] C. Borst, T. Wimbock, F. Schmidt, M. Fuchs, B. Brunner, F. Zacharias, P. R. Giordano, R. Konietschke, W. Sepp et al., "Rollin'Justin - Mobile Platform with Variable Base," in IEEE International Conference on Robotics and Automation (ICRA). IEEE, 2009.

[30] A. Dietrich, Whole-Body Impedance Control of Wheeled Humanoid Robots, ser. Springer Tracts in Advanced Robotics. Springer International Publishing, 2016.

[31] N. Y. Lii, A. Schiele, D. Leidner, P. Birkenkampf, R. Bayer, B. Pleintinger, A. Meissner, and B. Andreas, "Simulating an Extraterrestrial Environment for Robotic Space Exploration: the METERON SUPVIS-Justin Telerobotic Experiment and The Solex Proving Ground," in 13th Symposium on Advanced Space Technologies for Robotics and Automation, Noordwijk, The Netherlands, 2015.

[32] T. Mueller, "Col-CC Voice System migration during on-going operations," in Proceedings of the Spaceops Conference, 2012.

[33] J. Brooke, "SUS-A quick and dirty usability scale," Usability Evaluation in Industry, vol. 189, no. 194, pp. 4-7, 1996.

[34] N. Y. Lii, D. Leidner, P. Birkenkampf, B. Pleintinger, B. Bayer, and T. Krueger, "Toward Scalable Intuitive Telecommand of Robots for Space Deployment with METERON SUPVIS Justin," in Symposium on Advanced Space Technologies for Robotics and Automation, 2017.

[35] J. Vogel, D. Leidner, A. Hagengruber, M. Panzirsch, and A. Dietrich, "Das Projekt SMiLE," in Cluster-Konferenz Zukunft der Pflege, 2018. 